\title{
High Speed Railway Environment Safety Evaluation Based on Measurement Attribute Recognition Model
}

\author{
Qizhou Hu, ${ }^{1}$ Ningbo Gao, ${ }^{1}$ and Bing Zhang ${ }^{2}$ \\ ${ }^{1}$ School of Automation, Nanjing University of Science \& Technology, Nanjing, Jiangsu 2100984, China \\ ${ }^{2}$ East China Jiaotong University, Nanchang, Jiangxi 330013, China \\ Correspondence should be addressed to Ningbo Gao; 916747600@qq.com
}

Received 20 July 2014; Revised 22 September 2014; Accepted 25 September 2014; Published 9 November 2014

Academic Editor: Yongjun Shen

Copyright ( 2014 Qizhou Hu et al. This is an open access article distributed under the Creative Commons Attribution License, which permits unrestricted use, distribution, and reproduction in any medium, provided the original work is properly cited.

\begin{abstract}
In order to rationally evaluate the high speed railway operation safety level, the environmental safety evaluation index system of high speed railway should be well established by means of analyzing the impact mechanism of severe weather such as raining, thundering, lightning, earthquake, winding, and snowing. In addition to that, the attribute recognition will be identified to determine the similarity between samples and their corresponding attribute classes on the multidimensional space, which is on the basis of the Mahalanobis distance measurement function in terms of Mahalanobis distance with the characteristics of noncorrelation and nondimensionless influence. On top of the assumption, the high speed railway of China environment safety situation will be well elaborated by the suggested methods. The results from the detailed analysis show that the evaluation is basically matched up with the actual situation and could lay a scientific foundation for the high speed railway operation safety.
\end{abstract}

\section{Introduction}

According to the high speed railway safety operation research carried out in the laboratory of Nanjing University of Science and Technology, the high speed railway operation failure directly caused by bad environments accounts for $29 \%$ from July 2011 to December 2012, and comparatively the speed railway accidents in severe weather take up $81.4 \%$ of the total ones at the same time. The above statistics thus give us a better understanding of the fact that the bad weather has significant effects on the high speed railway safety operation.

In China, the current researches of environment impact on high speed railway can be mainly divided into the following two categories: first, the macrodisaster emergency prediction and warning system design and second, the microenvironmental factors impact mechanism analysis. As to the first one, Sun et al., Wang et al., and Tao et al. have outlined some key problems of high speed railway environment safety, such as alarm threshold, the layout of monitoring points, train controlling mode, and the basic component of high speed railway warning system [1-3]. Xiao et al., Calle-Sánchez et al., and Wang et al. also made an analysis of the potential factors which caused railway disaster from the following four aspects: personnel, equipment, management, and environment [46]. And Miyoshi and Givoni introduced analytic hierarchy process to set up railway environmental risk assessment system [7]. In the aspect of environmental factors impact mechanism, Zhou and Shen, Ling et al., and Lee et al. have made a specific discussion of such impact mechanism such as earthquake, wind, and other disasters in high speed railway from the view of engineering construction [8-10].

The comparison of the studies from abroad and home reveals that the researches of the high speed railway environment safety have been repeatedly carried out in an extremely earlier time and have been carefully studied by a lot of foreign researchers. Many countries have built up their own efficient high speed railway disaster warning system such as the Hokkaido and Shinkansen disaster warning system in Japan, which leads many other countries to conduct the earthquake prediction. For instance, France is now in possession of its Mediterranean earthquake monitoring system and Germany owns high speed railway disaster prevention system. Though the disaster monitoring systems of JingJingtang, Fuxia, and Wuguang have been already built in China, Zhang and Zeng 
TABLE 1: High speed railway mechanism analysis of environmental impact factors.

\begin{tabular}{ll}
\hline $\begin{array}{l}\text { Environmental } \\
\text { factor }\end{array}$ & Mechanism \\
\hline Rainfall & (i) Raining is the foremost factor that is easily causing line fault. Additionally, the current flow will emerge between \\
the pantograph and overhead line systems of the high speed railway when it comes to a heavy rainy day and the train \\
power supply will also be consequently influenced. \\
(ii) Rainfall can cause mountain soil landslides that will directly lead to the abnormal operation of the train.
\end{tabular}

contend that all the systems can be still well improved on the basis of the original ordinary railway disaster warning system [11] because there is a certain gap between foreign and China's high speed railway disaster warning systems after a relatively fair comparison.

Through the comparison of present researches between domestic and foreign, we can find that the domestic high speed railway disaster prevention is now in a transition from theory to practice, while foreign high speed railway disaster prevention system has been at a relatively perfect stage. Therefore, it is an urgent mission for the domestic researchers to make an intensive effort to the theory research of high speed railway disaster protection and system construction process so as to promote China high speed railway operating safety level.

\section{High Speed Railway Environmental Impact Evaluation Indexes}

2.1. High Speed Railway Index System of Environmental Impacts. The operational problems of the high speed railway are mainly caused by such uncertain factors as raining, thundering and lightning, horizontal wind, earthquake, and so forth, whose degree of intensity will directly decide the degree of danger posing to the high speed railway operation safety. The analysis of the characteristics of various environmental factors in the process of high speed railway operation in recent years and the conclusion of the mechanism of different environmental factors on high speed railway safe operation are presented in Table 1.

Besides the six factors listed in Table 1, problems in the high speed railway are also being influenced by debris flow and water and rock burst. However, given the complexity of geological conditions and the difficulty of data acquisition, we only use average annual rainfall, average annual maximum lightning density, annual disaster monsoon winds, average disasters incidence of monsoon, average magnitude grade, average incidence of earthquakes, average annual maximum snow depth, average highest temperature, and average minimum temperature as the environment factor evaluation index, which are shown in Figure 1.

It is necessary to be mentioned that the usual climate environment will not exert any influence upon the operation of high speed railway, except typhoon, sandstorm, blizzard, and earthquakes, while high or low temperatures have significant influence on the operation of high speed railway. Therefore, with the exclusive of the average rainfall in Figure 1, other factors represent the extreme climate environment. Each environmental factor evaluation index calculation formula and specification is shown in the following equations.

Average annual rainfall level is

$$
\mathrm{AAR}=\sum_{i=1}^{N} \frac{\mathrm{RF}_{i}}{N},
$$

where $\mathrm{RF}_{i}$ is the maximum rainfall in the $i$ th year $(\mathrm{mm})$ and is the number of the years.

Maximum lightning density is

$$
\operatorname{MLD}=\sum_{j=1}^{N} \frac{\mathrm{LH}_{j}}{(N \times \text { area })},
$$

where $\mathrm{LH}_{j}$ is the thunder lightning happening in certain region in the $j$ th year (time) and area is the area of a city or region $\left(\mathrm{m}^{2}\right)$.

Disasters wind speed is

$$
\mathrm{AWS}=\sum_{k=1}^{W_{n}} \frac{\mathrm{DWS}_{k}}{W_{n}},
$$




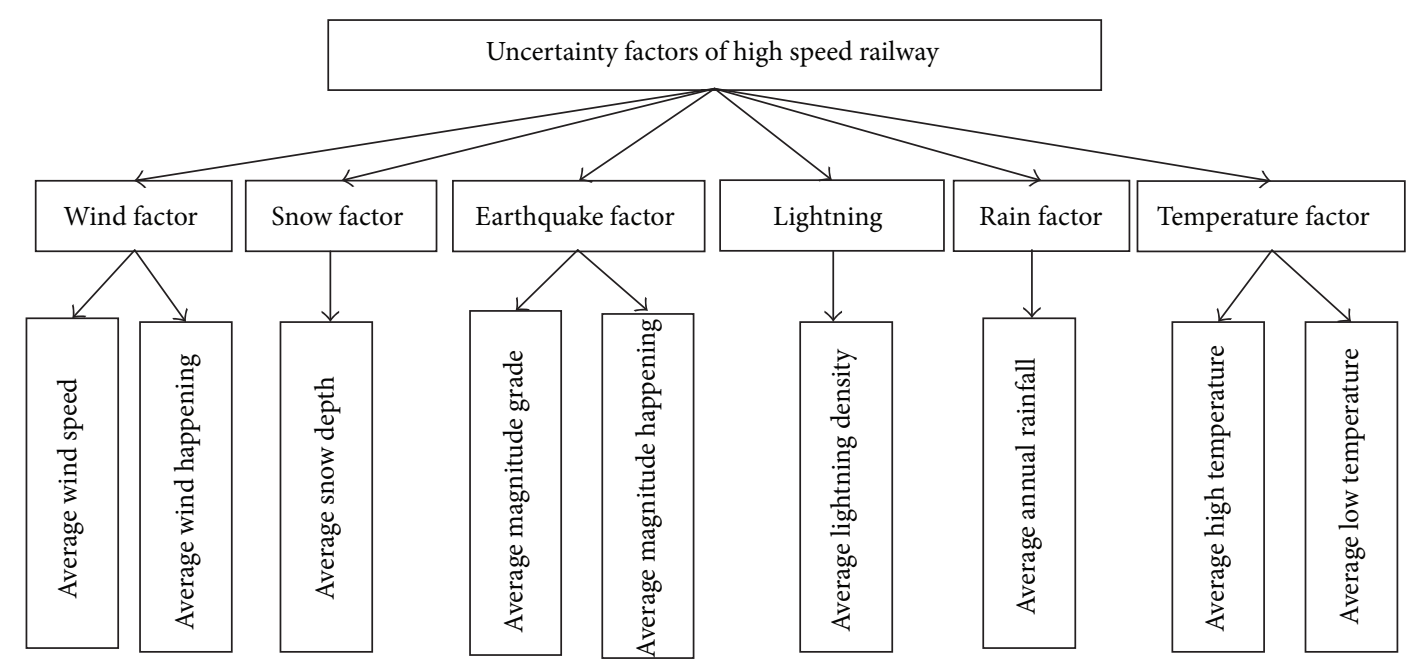

FIGURE 1: High speed railway environmental impact evaluation indexes system.

TABLE 2: Japanese Shinkansen winds threshold.

\begin{tabular}{lccc}
\hline Wind scale & Wind speed $(\mathrm{m} / \mathrm{s})$ & The impact with no wind-break wall & The impact with wind-break wall \\
\hline $8.0-9.0$ & $20-25$ & The train speed under $160 \mathrm{~km} / \mathrm{h}$ & No speed limit \\
$9.0-10.4$ & $25-30$ & The train speed under $70 \mathrm{~km} / \mathrm{h}$ & The train speed under $160 \mathrm{~km} / \mathrm{h}$ \\
$10.4-12.5$ & $30-35$ & Off-stream & The train speed under $70 \mathrm{~km} / \mathrm{h}$ \\
Above 12.5 & Above 35 & Off-stream & Off-stream \\
\hline
\end{tabular}

where DWS $\mathrm{D}_{k}$ is the speed of the $k$ th disaster wind $(\mathrm{m} / \mathrm{s})$ and area is the area of a city or region $\left(\mathrm{Km}^{2}\right)$.

Average wind happening is

$$
\mathrm{AWH}=\frac{W_{n}}{N}
$$

where $W_{n}$ is the total times of the disaster wind happening (time).

Average magnitude grade is

$$
\mathrm{AMG}=\sum_{l=1}^{D_{n}} \frac{\mathrm{MG}_{l}}{D_{n}},
$$

where $\mathrm{MG}_{l}$ is the magnitude of the $l$ th earthquake (degree) and $D_{n}$ is the total times of the earthquake (time).

Average magnitude happening is

$$
\mathrm{AWH}=\frac{D_{n}}{N}
$$

Average high and low temperature are

$$
\begin{aligned}
& \mathrm{AHT}=\sum_{p=1}^{N} \frac{\operatorname{Max} T_{p}}{N}, \\
& \mathrm{ALT}=\sum_{p=1}^{N} \frac{\operatorname{Min} T_{p}}{N},
\end{aligned}
$$

where $\operatorname{Max} T_{p}$ is the highest temperature in the $p$ th year $\left({ }^{\circ} \mathrm{C}\right)$ and Min $T_{p}$ is the lowest temperature in the $p$ th year $\left({ }^{\circ} \mathrm{C}\right)$.
Average snow depth is

$$
\mathrm{ASD}=\sum_{r=1}^{N} \frac{\operatorname{Max} \mathrm{SD}_{r}}{N},
$$

where $\mathrm{Max} \mathrm{SD}_{r}$ is the deepest depth in the $r$ th year $(\mathrm{cm})$.

\subsection{Demarcation of the Environmental Climate Factor Affected Threshold (Modify)}

2.2.1. Threshold under Horizontal Wind Influence. The representative research about the effects of horizontal wind on high speed railway train running is conducted preciously in Japan, which calculates the horizontal wind velocity under the condition of critical capsize under different running speed by wind tunnel experiment and takes the critical wind speed as the threshold of Shinkansen disaster warning (Table 2). China's high speed railway line train CRH series are characterized by the similar features with those of Japanese train in the shape and the axle load. Therefore, the Japanese Shinkansen warning horizontal wind speed is adopted as the influencing factors of high speed railway in our country horizontal wind threshold.

2.2.2. Threshold under Earthquake Influence. In terms of the research results at home and abroad, the calculation of earthquake alarm threshold (EAT) of high speed railway can be referred to as the following formula (9):

$$
\mathrm{EAT}=\frac{A}{D},
$$


TABLE 3: Earthquake magnitude threshold of high speed railway ( $D$ takes 2.55).

\begin{tabular}{|c|c|c|c|c|c|}
\hline Rank & Very serious & Serious & General & Slight & No effect \\
\hline Train lateral acceleration $(A)$ & $240 \mathrm{Gal}$ & $180 \mathrm{Gal}$ & $120 \mathrm{Gal}$ & $60 \mathrm{Gal}$ & $0 \mathrm{Gal}$ \\
\hline Earthquake magnitude (EAT) & $>5.2$ & 4.8 & 4.4 & 3.9 & $<3.9$ \\
\hline
\end{tabular}

TABLE 4: The annual rainfall threshold of high speed railway.

\begin{tabular}{|c|c|c|c|c|c|}
\hline Rank & Very serious & Serious & General & Slight & No effect \\
\hline Annual rainfall & $>2970 \mathrm{~mm}$ & $1980 \mathrm{~mm}$ & $900 \mathrm{~mm}$ & $600 \mathrm{~mm}$ & $<600 \mathrm{~mm}$ \\
\hline
\end{tabular}

where $A$ is the maximum lateral acceleration threshold ensuring that the normal operation of the train can withstand without orbit $(\mathrm{Gal}), D$ is the maximum dynamic response coefficient of various structures of railway under different seismic wave excitation, and suggestive value is 2.55 .

Researches show that when

case $A \geq 120 \mathrm{Gal}$, the train begins to pour;

case $A \geq 240 \mathrm{Gal}$, the train will completely overturn.

Therefore, we define $A=240 \mathrm{Gal}$ and $A=120 \mathrm{Gal}$ as the threshold of strong impact and general impact on the safe operation of the high speed railway train. And the earthquake magnitude threshold of high speed railway operation is calculated by different value method, which is shown in Table 3.

2.2.3. Threshold under Rain Influence. Domestic railway department limits the train running speed based on the size of the rain.

If the rain runs moderately which lasts 12 (or 24) hours and the rainfall capacity arrives at $10.0 \mathrm{~mm}-22.9 \mathrm{~mm}$ (17 mm-37.9 mm), its speed should be reduced.

If the rain runs in a heavy rainy day which lasts 12 (or 24) hours, and the rainfall capacity reaches $23.0 \mathrm{~mm}-49.9 \mathrm{~mm}$ $(33.0 \mathrm{~mm}-74.9 \mathrm{~mm})$, the railway lines are supposed to be blocked and the train operation is supposed to be prohibited.

For the sake of dimensional consistency, we can turn the hour rainfall volume into annual rainfall volume by the following method: it is universal knowledge that our country's rain season will experience a period of 3 months that can be calculated by 12 rainfall times; thus, we categorize the annual rainfall volume into $900 \mathrm{~mm}, 1980 \mathrm{~mm}$, and $2970 \mathrm{~mm}$, respectively, as the moderate rainfall city, heavy rainfall city, and the storm rainfall city. Accordingly, we can calculate rainfall threshold effects on high speed railway compared with the provisions of the railway departments in Table 4.

2.2.4. Other Environmental Factors Threshold. The current theoretical researches both at home and abroad pay less attention to the lightning, snowing, temperature, and snowfall which will definitely bring some influences on the characteristics of the high speed railway operations. Because it is difficult to set up a uniform standard to measure the factors, experts suggest that the reference value and the method of combining qualitative analysis can be employed to determine what degree of lightning, snow, and temperature influencing the high speed rail threshold. The environment impact assessment index of high speed railway can be discriminated as in Table 5.

\section{High Speed Railway Environmental Impacts Attribute Recognition Model}

Attribute recognition model is in essence the problems of multidimensional space between sample and attribution, which is proposed by professor Cheng and has been widely used in evaluation and classification. The sample space $X=$ $\left\{x_{1}, x_{2}, x_{3}, \ldots, x_{31}\right\}$ has been calculated in 31 provinces and autonomous regions in our country, among which each has been given nine high speed rail environmental impact indexes as $I_{j}(j=1,2, \ldots, 9)$, and the $j$ th environmental impact assessment index value in the $i$ th region is expressed as $x_{i j}(i=1,2, \ldots, 31 ; j=1,2, \ldots, 9) . F$ is defined on a sample space $X$ ordered split sets, where the environmental impact is divided into five progressive ways as serious, severe, moderate, mild model, and no effect. An ordered set of split is defined as $F=\left\{C_{1}, C_{2}, C_{3}, C_{4}, C_{5}\right\}$, which is in accordance with the relationship as $C_{1}>C_{2}>C_{3}>C_{4}>C_{5}$. Each ordered set is then to be split into a collection of environmental evaluation threshold segmentation classes. To make a clear illustration of the ordered stripe set, a standard form has been set up as follows:

$$
\begin{aligned}
& \begin{array}{lllll}
C_{1} & C_{2} & C_{3} & C_{4} & C_{5}
\end{array} \\
& I_{1} \quad \mid \begin{array}{lllll}
a_{11} & a_{12} & a_{13} & a_{14} & a_{15}
\end{array} \\
& \begin{array}{llllll}
I_{2} & a_{21} & a_{22} & a_{23} & a_{24} & a_{25}
\end{array} \text {, } \\
& \begin{array}{c|ccccc}
\vdots & \vdots & \vdots & \vdots & \vdots & \vdots \\
I_{9} & a_{91} & a_{92} & a_{93} & a_{94} & a_{95}
\end{array} \mid
\end{aligned}
$$

where $a_{i j}(i=1,2, \ldots, 9 ; j=1,2,3,4,5): a_{i 1}>a_{i 2}>a_{i 3}>$ $a_{i 4}>a_{i 5}$.

The value of the sample properties has attributes characterized by a sample $X_{i}$ and expressed as $u_{i k}=u\left(u_{i} \epsilon\right.$ $C_{k}$ ), among which the measurement function is the core of attribute recognition model. $\mathrm{Hu}$ et al., Yan, and Xiao et al. make an analysis of the usual linear discriminated function, whose accuracy is less than that of a nonlinear function. Therefore, the recent researches have found that the normal distribution function is used much more frequently, while other nonlinear functions are often being regarded as an attribute identification measure function [12-14]. However, the normal distribution function as a measure function has 
TABLE 5: High speed railway environment impact assessment index discrimination safety threshold.

\begin{tabular}{|c|c|c|c|c|c|c|}
\hline Environment & Evaluation Index & $\begin{array}{l}\text { Particularly serious } \\
\qquad C_{1}\end{array}$ & $\begin{array}{c}\text { Serious } \\
\mathrm{C}_{2} \\
\end{array}$ & $\begin{array}{c}\text { Medium } \\
\mathrm{C}_{3}\end{array}$ & $\begin{array}{c}\text { Slight } \\
C_{4} \\
\end{array}$ & $\begin{array}{c}\text { No effect } \\
C_{5} \\
\end{array}$ \\
\hline \multirow{2}{*}{ Cross wind } & $X_{1}(\mathrm{~m} / \mathrm{s})$ & $>30$ & $25-30$ & $15-25$ & $5-15$ & $0-5$ \\
\hline & $X_{2}$ (time) & $>3$ & $2-3$ & $1-2$ & $0.5-1.0$ & $0-0.5$ \\
\hline Snowfall & $X_{3}(\mathrm{~cm})$ & $>30$ & $22-30$ & $17-22$ & $9-17$ & $0-9$ \\
\hline \multirow{2}{*}{ Earthquake } & $X_{4}$ (level) & $>5.2$ & $4.8-5.2$ & $4.4-4.8$ & $3.9-4.4$ & $0-3.9$ \\
\hline & $X_{5}$ (time) & $>1.0$ & $0.6-1.0$ & $0.3-0.6$ & $0.1-0.3$ & $0-0.1$ \\
\hline Lightning & $X_{6}\left(\right.$ time $\left./ \mathrm{km}^{2}\right)$ & $>55$ & 45-55 & $30-45$ & 15-30 & $0-15$ \\
\hline Rainfall & $X_{7}(\mathrm{~mm})$ & $>2970$ & $1980-2970$ & $900-1980$ & $600-900$ & $0-600$ \\
\hline \multirow{2}{*}{ Temperature } & $X_{8}\left({ }^{\circ} \mathrm{C}\right)$ & $>64$ & $48-64$ & $35-48$ & $25-35$ & $10-25$ \\
\hline & $X_{9}\left({ }^{\circ} \mathrm{C}\right)$ & $<-20$ & $-10--20$ & $-10-0$ & $0-5$ & $5-10$ \\
\hline
\end{tabular}

$X_{1}$ : average disaster annual wind speed.

$X_{2}$ : the annual incidence of disasters monsoon.

$X_{3}$ : annual maximum snow depth.

$X_{4}$ : the average magnitude level.

$X_{5}$ : average annual rate of earthquake occurrence.

$X_{6}$ : annual lightning density.

$X_{7}$ : annual maximum rainfall.

$X_{8}$ : average annual maximum temperature.

$X_{9}$ : average annual minimum temperature.

its shortcomings because data should be standardized before handling bias and the separated index weights should also be determined. What is more, the last attribute recognition result is relative.

However, there is no certain way to evaluate the relative importance of objective indicators in a fairly way. The essence of attribute recognition is to determine the attributes space similarity and methods used to calculate the spatial distance are Euclidean distance, Ming distance, and Mahalanobis distance. Todeschini et al. and Kayaalp and Arslan assert that the Mahalanobis distance has the advantages of weakening the correlation between impact indicators and automatic weight in the index calculation based on data changes $[15,16]$.

Therefore, in order to compensate for normal function, we use Mahalanobis distance as the measurement function to build the attribute recognition model.

Step 1 (Mahalanobis distance between sample and attribute class calculations). Assuming the sample $X_{i}$ has been an area of environment evaluation, the sample Mahalanobis distance with the attribute class $C_{k}$ is

$$
d_{i k}=\sqrt{\left(X_{i}-C_{k}\right) \Sigma_{i k}^{-1}\left(X_{i}-C_{k}\right)^{T}},
$$

where $X_{i}=\left(x_{i 1}, x_{i 2}, \ldots, x_{i 9}\right)$, representing the $i$ th region environment factor evaluation vector, and $C_{k}=\left(a_{k 1}\right.$, $\left.a_{k 2}, \ldots, a_{k 9}\right)$, representing each classification criteria value of environmental factors on the properties class $k$ vector. $\Sigma_{i k}=$ the covariance matrix between $X_{i}$ and $C_{k}$ is

$$
\Sigma_{i k}=\left[\begin{array}{cccc}
\operatorname{Cov}\left(x_{i 1}, a_{k 1}\right) & \operatorname{Cov}\left(x_{i 1}, a_{k 2}\right) & \ldots & \operatorname{Cov}\left(x_{i 1}, a_{k 9}\right) \\
\operatorname{Cov}\left(x_{i 2}, a_{k 1}\right) & \operatorname{Cov}\left(x_{i 2}, a_{k 2}\right) & \ldots & \operatorname{Cov}\left(x_{i 2}, a_{k 9}\right) \\
\ldots & \ldots & \ldots & \ldots \\
\operatorname{Cov}\left(x_{i 9}, a_{k 1}\right) & \operatorname{Cov}\left(x_{i 9}, a_{k 2}\right) & \ldots & \operatorname{Cov}\left(x_{i 9}, a_{k 9}\right)
\end{array}\right],
$$

where $\operatorname{Cov}(x, y)=E[(x-E(x))(y-E(y))]$.
Step 2 (standard attribute measurement value calculations). Generally, the greater the similarity of Mahalanobis distance, the smaller the measurement value. Therefore, assuming that Mahalanobis distance between area $X_{i}$ and attribute class $C_{k}$ has been derived $d_{i k}$, the standard attribute measurement value is

$$
u_{i k}=\frac{1 / d_{i k}}{\sum_{j=1}^{5}\left(1 / d_{i j}\right)}
$$

Step 3 (sample class attribute recognition). Class attribute identification is in accordance with the confidence value $\lambda$ :

$$
\text { If } k_{i}=\min \left\{k: \sum_{l=1}^{k} u_{i l} \geq \lambda, k=5,4,3,2,1\right\}
$$

Then $X_{i}$ Can be considered as class $C_{k}$,

where $\lambda$ normal circumstances take $0.6 \leq \lambda \leq 0.7$.

Step 4 (security score calculations). Assuming each evaluation category $C_{k}$ corresponding score of $q_{k}$, then the combined attribute security score is

$$
S_{i}=\sum_{k=1}^{4} u_{i k} q_{k}
$$

\section{Case Studies}

4.1. Chinese Regions Environment Overview. Five domestic environmental factors such as rainfall, lightning, wind, temperature, and earthquake in recent years are collected from 2002 to 2012 as the basic assessments data [17] as is shown in Table 6. (The data of rain factor is summary of annual average rainfall in various regions, the data of thunder and lightning factors comes from various regions' monitoring reports, and 
TABLE 6: Chinese regional environment situation in recent years from 2002 to 2012.

\begin{tabular}{|c|c|c|c|c|c|c|c|c|c|}
\hline \multirow{2}{*}{ Region } & \multicolumn{9}{|c|}{ Index } \\
\hline & $X_{1}$ & $X_{2}$ & $X_{3}$ & $X_{4}$ & $X_{5}$ & $X_{6}$ & $X_{7}$ & $X_{8}$ & $X_{9}$ \\
\hline Beijing & 0 & 0 & 22.5 & 0 & 0 & 13.61 & 498.96 & 26.86 & -2.75 \\
\hline Tianjin & 41.67 & 0.11 & 19.7 & 0 & 0 & 8.6 & 504.6 & 26.84 & -3.71 \\
\hline Hebei & 47.92 & 0.22 & 20 & 7.8 & 0.03 & 29.97 & 544.97 & 27.48 & -1.77 \\
\hline Shanxi & 0 & 0 & 21 & 0 & 0 & 27.41 & 443.46 & 24.48 & -5.11 \\
\hline Inner Mongolia & 0 & 0 & 17 & 0 & 0 & 0.6 & 373.29 & 23.56 & -10.87 \\
\hline Liaoning & 41.67 & 0.11 & 25 & 7.3 & 0.02 & 8.75 & 701.63 & 24.3 & -12.01 \\
\hline Jilin & 26.39 & 0.11 & 27 & 0 & 0 & 9.25 & 598.41 & 23.38 & -14.6 \\
\hline Heilongjiang & 0 & 0 & 34 & 0 & 0 & 15.44 & 498.9 & 23.24 & -16.9 \\
\hline Shanghai & 32.99 & 0.89 & 8 & 0 & 0 & 17.176 & 1092.41 & 29.39 & 4.75 \\
\hline Jiangsu & 35.14 & 1.11 & 22 & 0 & 0 & 40.25 & 1164.72 & 28.81 & 2.95 \\
\hline Zhejiang & 39.22 & 2.78 & 6 & 0 & 0 & 76 & 1276.82 & 29.95 & 4.84 \\
\hline Anhui & 36.87 & 1.22 & 15 & 0 & 0 & 35.75 & 1057.21 & 28.74 & 2.76 \\
\hline Fujian & 39.56 & 3.22 & 4 & 0 & 0 & 35.3 & 1355.53 & 29.81 & 11.3 \\
\hline Jiangxi & 38.8 & 1.78 & 12 & 0 & 0 & 35 & 1500.14 & 30.13 & 5.53 \\
\hline Shandong & 33.8 & 0.33 & 17 & 0 & 0 & 32.5 & 820.57 & 26.98 & -1.21 \\
\hline Henan & 42.13 & 0.33 & 19 & 0 & 0 & 30.67 & 724.81 & 27.29 & 1.4 \\
\hline Hubei & 40.67 & 0.78 & 17 & 0 & 0 & 26.72 & 1210.48 & 29.83 & 4.38 \\
\hline Hunan & 35.52 & 0.78 & 16.4 & 0 & 0 & 29 & 1276.44 & 29.96 & 8.39 \\
\hline Guangdong & 33.93 & 4.11 & 0 & 0 & 0 & 48.25 & 1805.49 & 29.78 & 13.16 \\
\hline Guangxi & 34.92 & 2.33 & 4 & 0 & 0 & 26.25 & 1189.73 & 28.3 & 14.49 \\
\hline Hainan & 31.74 & 2.22 & 0 & 0 & 0 & 38.75 & 1780.62 & 29.06 & 14.07 \\
\hline Chongqing & 0 & 0 & 3.7 & 0 & 0 & 23.58 & 1065.61 & 29.53 & 6.89 \\
\hline Sichuan & 0 & 0 & 4.2 & 7.44 & 0.1 & 57.256 & 843.16 & 25.96 & 5.98 \\
\hline Guizhou & 43.06 & 0.11 & 4.5 & 0 & 0 & 31.75 & 989.78 & 23.19 & 3.52 \\
\hline Yunnan & 35.19 & 0.33 & 0 & 7.33 & 0.1 & 27.21 & 878.28 & 21 & 9.62 \\
\hline Tibet & 0 & 0 & 52 & 0 & 0 & 0.29 & 453.12 & 17.31 & 0.62 \\
\hline Shanxi & 15 & 2 & 19 & 0 & 0 & 15.46 & 611.11 & 27.61 & 0.36 \\
\hline Gansu & 12.4 & 2.22 & 18 & 6.6 & 0.02 & 0.36 & 271.74 & 22.46 & -5.06 \\
\hline Qinghai & 32 & 3.56 & 15 & 6.9 & 0.02 & 0.42 & 442.9 & 17.48 & -7.75 \\
\hline Ningxia & 4.72 & 1.89 & 17.9 & 0 & 0 & 4.77 & 175.34 & 24.31 & -7.38 \\
\hline Xinjiang & 46 & 4.67 & 46 & 7.1 & 0.05 & 0.25 & 309.61 & 24.19 & -12.9 \\
\hline
\end{tabular}

the data of wind factor represents the influence extent by monsoon in various regions.)

The program of MATLAB is employed to work out the estimation. The specific method is made by 31 districts samples and each has 9 indexes. Then we constitute the sample matrix $R_{31 \times 9}$. There are five characteristics consisting of particularly serious, severe, moderate, mild, and no effect, whose intermediate values will be made up of attribute matrix $R_{5 \times 9}$; that is,

$$
R_{5 \times 9}=\left[\begin{array}{ccccc}
35.0 & 32.5 & 27.5 & 22.5 & 10.0 \\
3.00 & 2.50 & 1.50 & 0.75 & 0.25 \\
30.0 & 26.0 & 19.5 & 13.0 & 4.50 \\
5.20 & 5.00 & 4.60 & 4.15 & 1.95 \\
1.00 & 0.80 & 0.45 & 0.20 & 0.05 \\
55.0 & 50.0 & 40.0 & 22.5 & 7.50 \\
2970 & 2475 & 1440 & 750 & 300 \\
64.0 & 56.0 & 41.5 & 30.0 & 17.5 \\
-20.0 & -15.0 & -5.00 & 2.50 & 7.50
\end{array}\right]
$$

Use the function pdist of MATLAB to work out the Mahalanobis distance between the districts sample and the attribute class:

$$
z=\operatorname{pdist}\left(R_{31 \times 9}, R_{5 \times 9}\right. \text {, "mahal"), }
$$

where $z$ is the Mahalanobis distance matrix between the sample and the attribute and mahal is representing the use of the function Mahalanobis distance to work out the distance of matrix.

Then make confidence level $\lambda=0.60$, and each of the area's environmental attribute recognition values and attribute classification can be obtained as that in Table 7.

The calculation results in the above table show that the environmental safety situation of Xinjiang, Sichuan, Heilongjiang, and Jilin belongs to serious category, which takes up $12.9 \%$. The situation in the Medium level areas accounts for $32.2 \%$, such as Heilongjiang, Hebei, Liaoning, Jiangsu, and Guangdong, and that of the 17 areas such as Beijing, Tianjin, Guizhou, Gansu, and other regions belongs to slight 
TABLE 7: Chinese regional environment impacts attribute recognition value of high speed railway.

\begin{tabular}{|c|c|c|c|c|c|c|}
\hline \multirow{2}{*}{ Region } & \multicolumn{6}{|c|}{ Value } \\
\hline & Particularly serious & Serious & Medium & Slight & No effect & Classification \\
\hline Xinjiang & 0.301 & 0.402 & 0.117 & 0.003 & 0.177 & \multirow{4}{*}{ Serious } \\
\hline Sichuan & 0.376 & 0.246 & 0.196 & 0.120 & 0.062 & \\
\hline Jilin & 0.303 & 0.363 & 0.146 & 0.082 & 0.106 & \\
\hline Heilongjiang & 0.269 & 0.342 & 0.215 & 0.140 & 0.034 & \\
\hline Hebei & 0.169 & 0.196 & 0.237 & 0.231 & 0.168 & \multirow{10}{*}{ Medium } \\
\hline Liaoning & 0.201 & 0.203 & 0.218 & 0.198 & 0.180 & \\
\hline Jiangsu & 0.177 & 0.209 & 0.228 & 0.211 & 0.174 & \\
\hline Zhejiang & 0.180 & 0.202 & 0.225 & 0.205 & 0.188 & \\
\hline Anhui & 0.166 & 0.202 & 0.234 & 0.221 & 0.177 & \\
\hline Jiangxi & 0.196 & 0.222 & 0.206 & 0.199 & 0.178 & \\
\hline Hubei & 0.179 & 0.210 & 0.221 & 0.216 & 0.175 & \\
\hline Hunan & 0.175 & 0.205 & 0.221 & 0.222 & 0.176 & \\
\hline Guangdong & 0.195 & 0.200 & 0.212 & 0.198 & 0.196 & \\
\hline Fujian & 0.194 & 0.211 & 0.195 & 0.200 & 0.200 & \\
\hline Beijing & 0.163 & 0.193 & 0.226 & 0.239 & 0.179 & \multirow{17}{*}{ Slight } \\
\hline Tianjin & 0.158 & 0.188 & 0.232 & 0.234 & 0.187 & \\
\hline Shanxi & 0.160 & 0.190 & 0.227 & 0.228 & 0.195 & \\
\hline Inner Mongolia & 0.178 & 0.200 & 0.202 & 0.212 & 0.208 & \\
\hline Shanghai & 0.173 & 0.203 & 0.215 & 0.224 & 0.185 & \\
\hline Hainan & 0.168 & 0.198 & 0.222 & 0.224 & 0.189 & \\
\hline Shandong & 0.162 & 0.196 & 0.234 & 0.222 & 0.186 & \\
\hline Henan & 0.150 & 0.184 & 0.247 & 0.237 & 0.182 & \\
\hline Guangxi & 0.151 & 0.181 & 0.222 & 0.248 & 0.199 & \\
\hline Chongqing & 0.180 & 0.201 & 0.208 & 0.223 & 0.187 & \\
\hline Guizhou & 0.162 & 0.187 & 0.202 & 0.206 & 0.244 & \\
\hline Yunnan & 0.153 & 0.177 & 0.201 & 0.229 & 0.239 & \\
\hline Tibet & 0.178 & 0.192 & 0.202 & 0.213 & 0.215 & \\
\hline Shaanxi & 0.155 & 0.187 & 0.235 & 0.245 & 0.178 & \\
\hline Gansu & 0.165 & 0.191 & 0.215 & 0.237 & 0.192 & \\
\hline Qinghai & 0.177 & 0.194 & 0.194 & 0.203 & 0.231 & \\
\hline Ningxia & 0.155 & 0.183 & 0.224 & 0.237 & 0.200 & \\
\hline
\end{tabular}

level, which accounts for $54.9 \%$. It is notable that, in addition to Sichuan, the high speed railway environment impacts in the serious level areas are mostly distributed in coastal areas and northern regions, while Chinese abdominal regions are mostly in the medium and light level (see Figure 2).

For further analysis, security score in the areas of the serious level should be calculated by means of grading criterion. All kinds of scores are clarified in Table 8.

The calculation results show that Sichuan has the lowest scores of 62.460 , followed by 63.280 in Heilongjiang and 63.489 in Xinxiang, and Yunnan has the highest score of 72.23 .

4.2. High Speed Railway Line Safety Environment Analysis. There are 25 high speed railway operational lines in our country currently, which constitute the total mileage of 10192 kilometers. Most of the high speed railways are located in southeast of China, where complex geological accidents such as landslip, earthquake, and other geological disasters take place frequently. The high speed railway environment safety situation is clearly illustrated in Table 9.

The Jinghu line, Fuxia line, and Huning line mainly go across regions of Beijing, Tianjin, Jinan, Nanjing, Shanghai, Hangzhou, and so on. Most of these regions are located in the medium impacted or light impacted areas where raining and storm happen frequently. Thus, we have to pay attention to the influence of heavy rain and storm.

The Wuguang line and Guangshengang line mainly go cross such cities as Guangzhou, Foshan, and others in Guangzhou. These cities are vulnerable to the typhoon from coastal regions, which will affect the progress of the high speed railway.

The Yiwan line, Suiyu line, and Dacheng line go cross Wanzhou, Suining, Shizishan, Chengdu, or other cities of Sichuan province. High speed railway in these areas will suffer seriously from the tough environment, and we should pay attention to prevent cost and loss from landslip and earthquake. 


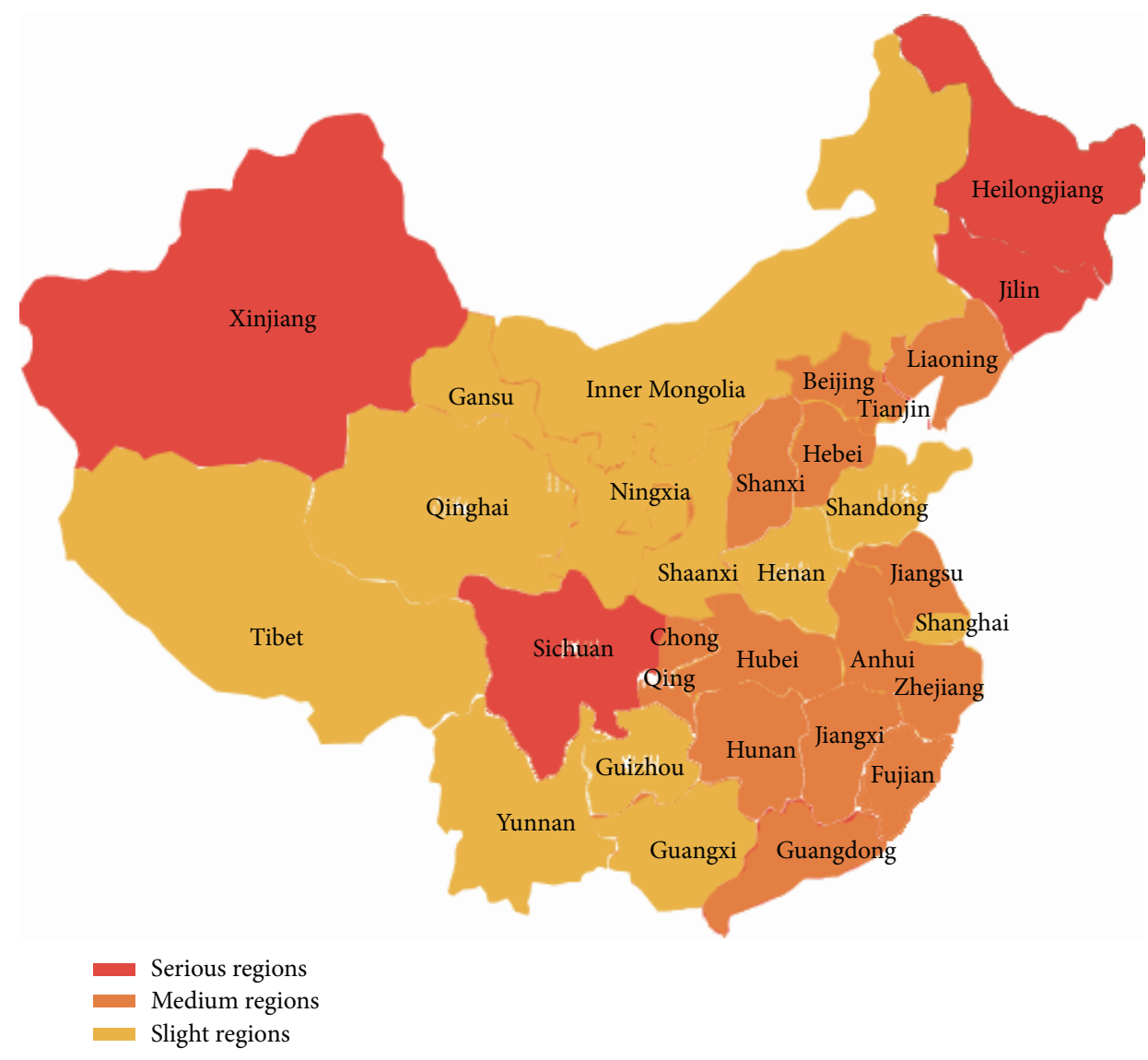

FIGURE 2: Chinese environment impacts of high speed railway distribution.

TABLE 8: The attribute recognition of high speed railway classification score.

\begin{tabular}{lccccc}
\hline Classification & No effect & Slight & Medium & Serious & Particularly serious \\
\hline Score & 90 & 80 & 70 & 60 & 50 \\
\hline
\end{tabular}

\section{Conclusions}

Firstly, the paper makes a detailed analysis of the impact from such environment factors as rainfall, earthquake, lightning, wind, and snow on the high speed railway safety mechanism. On the basis of the analysis, the evaluation index system of safety has been established and the threshold of high speed railway environmental safety has been calibrated by citing the results of domestic and abroad. At last, the high speed railway uncertain safety attribute recognition model is created based on the Mahalanobis distance with the features of dimensionless and weak effect correlation, which simplifies the comprehensive calculation process.

Secondly, the examples of China's 31 provinces and regions in the paper are selected to make the data of the high speed railway environmental safety much more convincing. The degree of danger is divided into five categories, among which the cities that the high speed railways pass in the serious category account for $16.1 \%$, those in the middle class account for $38.7 \%$, those in the mild category account for
$38.7 \%$, and those in the no effect category account for $6.51 \%$. It deserves our attention that cities of Xinjiang, Sichuan, Guangdong, Heilongjiang, and Liaoning belong to the serious category, whose evaluation results are basically consistent with the environmental characteristics. And the results have a certain theoretical reference for the " 135 " planning of high speed railway operation safety in Xinjiang and other areas.

At last, the analysis of the high speed railway environmental safety is directed to the aspect of weather, geology, and other factors. However, considering the complexity of data acquisition, the high speed railway evaluation index has its own drawbacks in this paper. It is needed to introduce more methods and factors into the evaluation of the high speed railway safety operation to facilitate the further researches.

\section{Conflict of Interests}

The authors declare that there is no conflict of interests regarding the publication of this paper. 
TABle 9: The environment impacts of high speed railway lines distribution.

\begin{tabular}{|c|c|c|c|}
\hline Lines & Serious environmental impact & Middle environment impact & Light environmental impact \\
\hline Jinhu & - & Nanjing, Jinan & Beijing, Tianjin, and Shanghai \\
\hline Hebang & - & Hefei, Bengbu & - \\
\hline Jinshi & - & Shijiazhuang & Beijing \\
\hline Wuguang & - & Wuhan, Changsha & Guangzhou, Foshan \\
\hline Guangshengang & - & - & Guangzhou, Shenzhen \\
\hline Yongtaiwen & - & - & Ningbo, Taizhou, and Wenzhou \\
\hline Wenfu & - & Wenzhou, Fuzhou & - \\
\hline Fuxia & - & Fuzhou, Xiamen, and Quanzhou & - \\
\hline Zhenxi & & - & Zhengzhou, Xi 'an \\
\hline Xibao & - & - & Xi ’an, Baoji \\
\hline Huhang & - & Jiaxing, Hangzhou & Shanghai \\
\hline Hewu & - & Nanjing, Hefei, and Wuhan & - \\
\hline Hanyi & - & Hankou, Zhijiang, and Yinchuan & - \\
\hline Hening & - & Hefei, Nanjing & - \\
\hline Jiaoji & - & - & Jinan, Qingdao \\
\hline Shitai & - & - & Shijiazhuang, Taiyuan \\
\hline Huning & - & Nanjing, Suzhou & Shanghai \\
\hline Yiwan & Wangzhou, Ensi & - & Yichang \\
\hline Yuli & - & Chongqing, Fuling & Liangwu \\
\hline Suiyu & Suining, Shizuishan & - & - \\
\hline Dacheng & Dazhou, Suining, and Chengdu & - & - \\
\hline
\end{tabular}

\section{Acknowledgments}

The authors are very grateful to the anonymous referees for their insightful and constructive comments and suggestions that have led to an improved version of this paper. The work also was supported by National Nature Science Funding of China (Project no. 51178157), The Basic Scientific Research Business Special Fund Project in Colleges and Universities (no. 2011zdjh29), National Statistical Scientific Research Projects (no. 2012LY150), "Blue Project" Projects in Jiangsu Province Colleges and Universities (no. 201211), and Youth Fund Projects in Jiangxi Province Department of Education (no. GJJ13314).

\section{References}

[1] L. Sun, H. Zhong, and G. Lin, "An overview of earthquake early warning systems for high speed railway and its application to Beijing-Shanghai high speed railway," World Earthquake Engineering, vol. 27, no. 3, pp. 14-16, 2011.

[2] L. Wang, Y. Qin, J. Xu, and L. Jia, "A fuzzy optimization model for high-speed railway timetable rescheduling," Discrete Dynamics in Nature and Society, vol. 2012, Article ID 827073, 22 pages, 2012.

[3] H. Tao, N. Hong-Xia, and F. Duo-Wang, "Speed contron for high-speed railway on multi-mode intelligent control and feature recognition," Telkomnika, no. 8, pp. 2069-2074, 2012.

[4] X. Xiao, L. Ling, and X. Jin, "A study of the derailment mechanism of a high speed train due to an earthquake," Vehicle System Dynamics, vol. 50, no. 3, pp. 449-470, 2012.

[5] J. Calle-Sánchez, M. Molina-García, J. I. Alonso, and A. Fernández-Durán, "Long term evolution in high speed railway environments: feasibility and challenges," Bell Labs Technical Journal, vol. 18, no. 2, pp. 237-253, 2013.

[6] H. Wang, W. Xu, F. Wang, and C. Jia, "A cloud-computingbased data placement strategy in high-speed railway," Discrete Dynamics in Nature and Society, vol. 2012, Article ID 396387, 15 pages, 2012.

[7] C. Miyoshi and M. Givoni, "The environmental case for the high-speed train in the UK: examining the London-Manchester route," International Journal of Sustainable Transportation, vol. 8, no. 2, pp. 107-126, 2014.

[8] L. Zhou and Z. Shen, "Progress in high-speed train technology around the world," Journal of Modern Transportation, vol. 19, no. 1, pp. 1-6, 2011.

[9] L. Ling, X. Xiao, and X. Jin, "Study on derailment mechanism and safety operation area of high-speed trains under earthquake," Journal of Computational and Nonlinear Dynamics, vol. 7, no. 4, Article ID 041001, 2012.

[10] K. S. Lee, J. K. Eom, J. Lee et al., “The preliminary analysis of introducing $500 \mathrm{~km} / \mathrm{h}$ high-speed rail in Korea," International Journal of Railway, vol. 6, no. 1, pp. 26-31, 2013.

[11] W. Zhang and J. Zeng, "A review of vehicle system dynamics in the development of high-speed trains in China," International Journal of Dynamics and Control, vol. 1, no. 1, pp. 81-97, 2013.

[12] Q.-Z. Hu, H.-P. Lu, and W. Deng, "Evaluating the urban public transit network based on the attribute recognition model," Transport, vol. 25, no. 3, pp. 300-306, 2010.

[13] M. Yan, "Multigranulations rough set method of attribute reduction in information systems based on evidence theory," Journal of Applied Mathematics, vol. 2014, Article ID 857186, 9 pages, 2014.

[14] Z. Xiao, W. Chen, and L. Li, "A method based on intervalvalued fuzzy soft set for multi-attribute group decision-making 
problems under uncertain environment," Knowledge and Information Systems, vol. 34, no. 3, pp. 653-669, 2013.

[15] R. Todeschini, D. Ballabio, V. Consonni, F. Sahigara, and P. Filzmoser, "Locally centred Mahalanobis distance: a new distance measure with salient features towards outlier detection," Analytica Chimica Acta, vol. 787, pp. 1-9, 2013.

[16] N. Kayaalp and G. Arslan, "A fuzzy bayesian classifier with learned mahalanobis distance," International Journal of Intelligent Systems, vol. 29, no. 8, pp. 713-726, 2014.

[17] http://cdc.cma.gov.cn/home.do. 

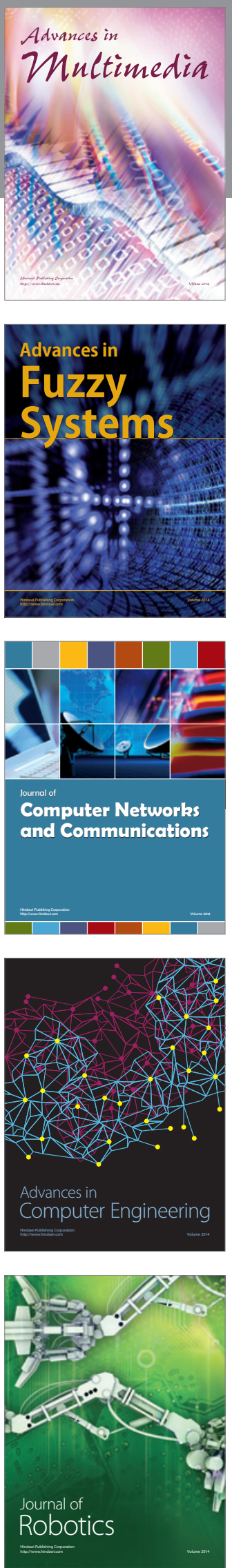

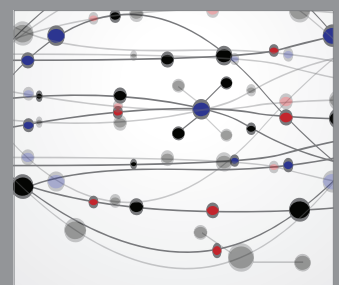

The Scientific World Journal
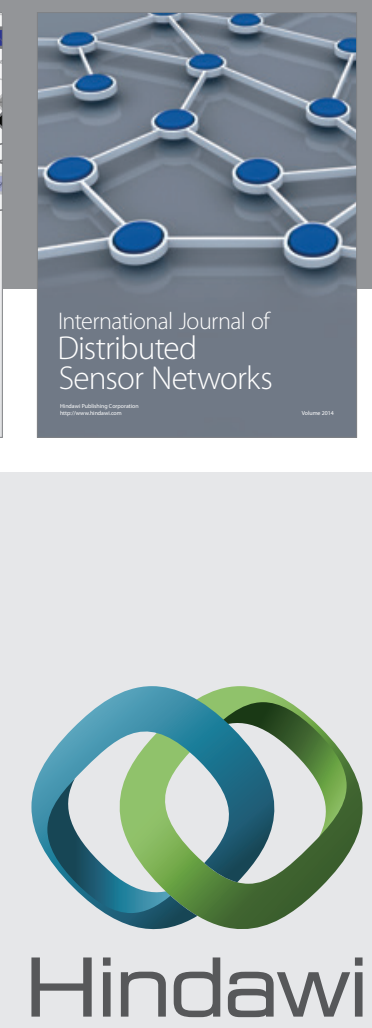

Submit your manuscripts at

http://www.hindawi.com
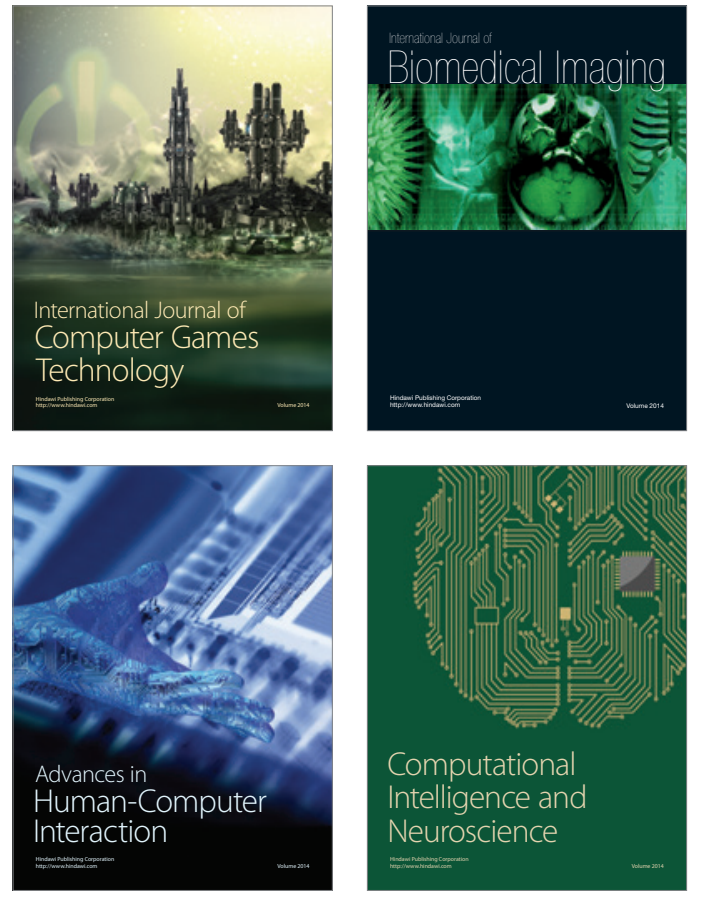
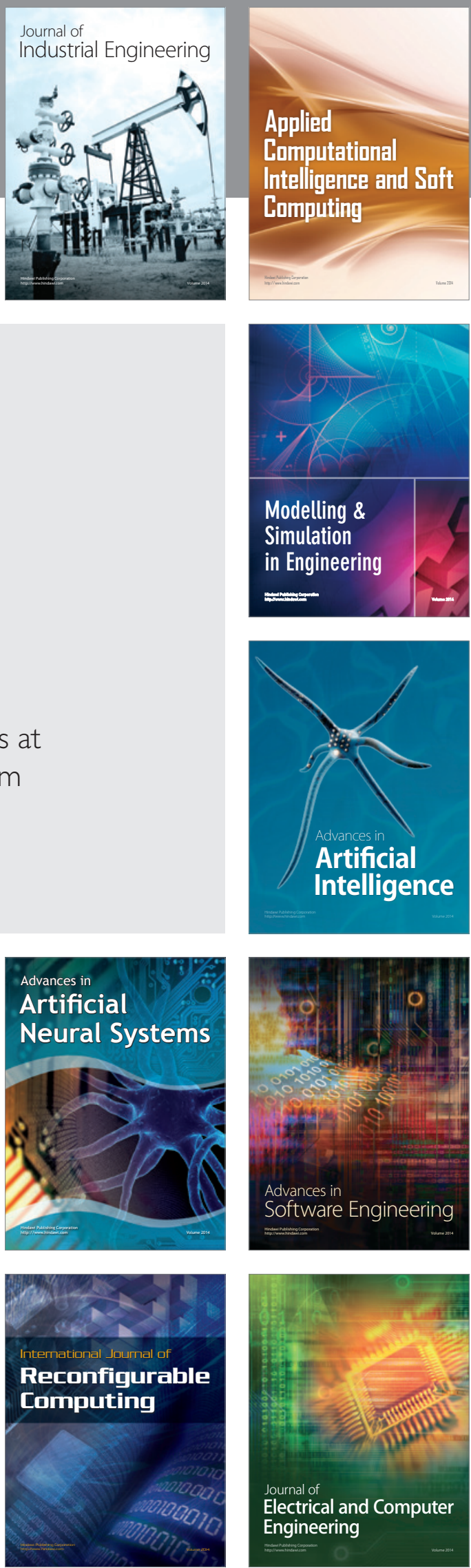